hep-th/9910082

\title{
Comments on Black Holes in String Theory
}

\author{
GARY T. HOROWITZ \\ Physics Department, University of California, Santa Barbara, CA 93106, USA
}

\begin{abstract}
A very brief review is given of some of the developments leading to our current understanding of black holes in string theory. This is followed by a discussion of two possible misconceptions in this subject - one involving the stability of small black holes and the other involving scale radius duality. Finally, I describe some recent results concerning quasinormal modes of black holes in anti de Sitter spacetime, and their implications for strongly coupled conformal field theories (in various dimensions).
\end{abstract}

October, 1999

To appear in the proceedings of the Strings '99 conference, Potsdam, Germany, July 1999. 


\section{Review}

This talk is divided into three parts. The first is a brief review of some of the key developments leading to our current understanding of black holes in string theory. This part will be very elementary, and not assume much knowledge of string theory. Next, I will try to clear up two misconceptions that I had until recently, and that I have seen in the literature. Finally, I will describe some recent work about black holes in anti de Sitter spacetime, and their implications for the approach to thermal equilibrium in strongly coupled conformal field theories.

Since supergravity is the low energy limit of string theory, the study of black holes begins by finding solutions to this theory with horizons. Actually, there are several supergravity theories that arise in string theory, starting with the ten and eleven dimensional theories. Since we are in higher dimensions, there are extended black holes, or black $p$ branes. The simplest solutions are products of $R^{p}$ with the $D-p$ dimensional Schwarzschild solution (where $D=10$ or 11), but more interesting solutions carry charge associated with a $p+2$ form. The rank is $p+2$ since the solution has $p$ spatial dimensions along the brane. Adding one for time and one for the radius in the transverse space, one finds that a sphere $S$ which surrounds the brane must have dimension $D-(p+2)$. The charge is then $Q \sim \int_{S}{ }^{*} F_{p+2}$. This charge can be nonzero even though there are no fundamental sources in supergravity, since all you need is nontrivial spacetime topology. This is exactly analogous the existence of charged black holes in Einstein-Maxwell theory without charged matter. The first charged black $p$-branes that were found almost ten years [1] ago assumed maximal symmetry, so all fields were a function of only one radial variable. These solutions depended on two parameters which were the mass $M$ (really mass per unit volume) and charge. Solutions existed only when $M$ and $Q$ satisfied a certain inequality. Since then, the number of black $p$-brane solutions has grown enormously as people have learned how to add multiple charges, rotation, traveling waves, etc. [2].

Unlike supergravity, string theory does have sources for many of these charges called D-branes [3]. The charge to mass ratio of these D-branes is exactly the same as the extremal limit of the black $p$-branes, so the latter can be interpreted as the gravitational field of the D-brane. At weak coupling, this gravitational field goes to zero and the low energy excitations of $N$ parallel D-branes are described by an $S U(N)$ gauge theory. The strongly coupled description of the same excited system should be a nonextreme black $p$-brane. By comparing the weak and strong coupling descriptions, one had the possibility 
of understanding black hole entropy by counting quantum states for the first time.

As an example, consider $N$ three-branes. To keep all quantities finite, it is convenient to compactify the directions along the brane into a three torus. Then, in the extreme limit, the area of the horizon goes to zero, which agrees with the fact that at zero excitation energy, the only state in the gauge theory is the ground state. To compare the entropies, we want to add energy to the system. Equivalently, we can consider nonzero temperature $T$. The effective coupling is $g N$ where $g$ is the string coupling constant. When $g N \ll 1$ the system is a weakly coupled $3+1$ dimensional gauge theory at temperature $T$. When $g N \gg 1$ one has a near extremal black three-brane at the same temperature. One can compare the entropies and find [4]

$$
S_{b h}=\frac{3}{4} S_{\text {gauge }}
$$

where $S_{b h}$ is the Bekenstein Hawking entropy of the black three-brane. So the gauge theory has roughly the right number of degrees of freedom to explain the entropy of near extremal black three-branes. The fact that they are not exactly the same was not a surprise. At the time this was first computed, it appeared that one had two different descriptions of the same system which were valid for different ranges of the parameter $g N$. They appeared to have no overlapping region of validity.

However, there were other situations where the entropies agreed exactly. These were obtained by looking at solutions with more than one charge. For example, suppose four dimensions of space are compactified on a small $T^{4}$. We can take $Q_{5}$ five-branes and wrap them around the compact dimensions to produce an effective string in six dimensions. One can then add $Q_{1}$ one-branes to this string. When $g^{2} Q_{1} Q_{5} \ll 1$, the low energy excitations are described by a $1+1$ dimensional conformal field theory [5]. When $g^{2} Q_{1} Q_{5} \gg 1$ the system is described by a black string in six dimensions. If one now adds a small amount of energy and compares the entropies, one finds complete agreement (for large charges) [6]

$$
S_{b h}=S_{c f t}
$$

Why is this working? For the special case where the momentum along the effective string is equal to the added energy, i.e., one excites only right moving modes, there is unbroken supersymmetry. The momentum along the string is like another charge, and the black string remains extremal. In this case, one can show that the number of supersymmetric states should not depend on the coupling. But the entropy turns out to agree even 
when supersymmetry is broken, e.g., when you excite equal amounts of left and right moving modes [7,8]. Even more importantly, the spectrum of Hawking radiation also agrees [9, 10.

The situation was clarified by Maldacena [11] who took a low energy limit which decoupled the excitations of the branes from the excitations of the strings off the branes. At strong coupling, this same limit corresponded to considering strings moving very close to the horizon of the black $p$-brane. In the cases of interest, the excitation of the branes is described by a conformal field theory (CFT), and the near horizon geometry of the extremal black $p$-brane is a product of anti de Sitter (AdS) space and a sphere. For example, in the case of the three brane, this geometry is $A d S_{5} \times S^{5}$ where the radii of curvature are equal. For the one-brane five-brane system, the near horizon geometry is $A d S_{3} \times S^{3} \times T^{4}$. Since the conformal field theory is well defined even at strong coupling, we obtain two different descriptions which are now valid for the same range of parameters. This lead Maldacena to his famous AdS/CFT correspondence. If one adds energy to the system, the spacetime is not exactly $\mathrm{AdS}$, but still approaches it asymptotically. So the correspondence says that string theory in spacetimes which asymptotically approach AdS times a sphere is completely described by a conformal field theory. There is growing evidence in support of this remarkable conjecture [12].

How does this explain the entropy results? For the case of the three-brane, it is easy to see from the field theory side why the entropy might change between weak and strong coupling. As you increase the coupling constant you add potential energy to each state and increase its energy, so the number of states for given total energy goes down. Similarly, from the gravity side one can understand the change in entropy as follows. The near horizon geometry of the near extremal solution is a black hole in AdS. As you lower the string coupling, the spacetime curvature increases in string units. This results in stringy corrections to the geometry, and hence corrections to the black hole entropy. In light of these effects, one would expect the weak coupling and strong coupling results to be related in a complicated way. The fact that they are related by a simple factor of $3 / 4$ is rather mysterious and still not understood.

In contrast, for the one-brane five-brane system, one has a $1+1$ dimensional CFT whose entropy depends only on the central charge. This can be computed exactly, and is independent of the coupling constant. On the gravity side, the near horizon geometry turns out to be the product of a three dimensional BTZ black hole [13] and $S^{3} \times T^{4}$. This 
is locally a space of constant curvature and probably does not receive string corrections as $g \rightarrow 0$ [14]. There are other systems where the entropy can be computed exactly without supersymmetry, including near extremal four dimensional black holes. But as far as I know, in all such cases the corresponding field theory is a $1+1$ dimensional CFT and the near horizon geometry is a space of constant curvature. (When there is unbroken supersymmetry, the entropy can be reproduced for a wider class of black holes, including higher order corrections to the Bekenstein Hawking entropy [15].)

In light of the AdS/CFT correspondence, we can begin to translate questions about black hole physics into questions about field theory. For example, the formation of a large black hole in AdS is not an exotic process in the CFT. It corresponds to the field theory evolution of a very special high energy state into a typical (approximately thermal) state. More importantly, the formation and evaporation of a small black hole in AdS should be described by the usual unitary evolution in the field theory.

\section{Misconceptions}

We now come to our first possible misconception, which involves small black holes in AdS. Let $r_{+}$denote the horizon radius, and $R$ denote the radius of AdS. The temperature of a black hole in AdS decreases with mass for $r_{+} \ll R$, but increases with mass for $r_{+} \gg R$. So large black holes have positive specific heat and are stable. Small black holes have negative specific heat and one often concludes that they are unstable and will evaporate. However, let us compare the entropy of a small black hole with the entropy of the same amount of energy in a thermal gas. We will consider the case of black holes in $A d S_{5} \times S^{5}$. Since the magnitude of the curvature on $S^{5}$ is the same as $A d S_{5}$, a gas of radiation will be effectively ten dimensional. Since the curvature of AdS acts like a confining box of side $R$, the gas has $S \sim T^{9} R^{9}$ and $E \sim T^{10} R^{9}$, so

$$
S_{\text {gas }} \sim(R E)^{9 / 10}
$$

A small black hole in $A d S_{5}$ which is uniform over the $S^{5}$ is unstable to localizing on the $S^{5}$ due to the Gregory-Laflamme instability [16]. So we should use ten dimensional black holes which have $S_{b h} \sim r_{+}^{8} / \ell_{p}^{8}$ (where $\ell_{p}$ is the ten dimensional Planck scale) and $E \sim r_{+}^{7} / \ell_{p}^{8}$, which implies $S_{b h} \sim E r_{+}$. Now let $R^{8} \sim N^{2} \ell_{p}^{8}$, so $N$ is a measure of how large the $S^{5}$ (or $A d S_{5}$ ) is in Planck units. (In the AdS/CFT correspondence, this is the same $N$ that appears in the group $S U(N)$, but since we are asking a pure supergravity question, we 
don't need to introduce any string theory or gauge theory quantities.) So the entropies will be equal when

$$
S_{b h} \sim \frac{N^{2} r_{+}^{8}}{R^{8}} \sim(R E)^{9 / 10} \sim\left(\frac{N^{2} r_{+}^{7}}{R^{7}}\right)^{9 / 10}
$$

This implies

$$
\frac{r_{+}}{R} \sim \frac{1}{N^{2 / 17}}
$$

which can be made arbitrarily small for large $N$. In other words, if $r_{+} / R>N^{-2 / 17}$, the black hole has more entropy than a gas in AdS. So its evaporation would violate the second law of thermodynamics. What happens?

If you fix the temperature, a small black hole with high temperature will simply absorb energy from the heat bath until it turns into a large black hole with the same temperature which is stable. But this is rather unphysical since its hard to connect a heat bath to AdS, and this is not the right boundary conditions when a black hole evaporates. One should instead fix the total energy, and consider a system consisting of both a black hole and radiation. It is clear that if you start with all the energy in the black hole and radiate a small amount $\epsilon, \delta S_{b h} \sim-\epsilon$ and $\delta S_{g} \sim \epsilon^{9 / 10}$. So $\delta S_{g}+\delta S_{b h}>0$ and you increase the total entropy by starting to radiate. This is a consequence of the negative specific heat. But to see the final outcome, we must maximize the total entropy for given energy. Let us divide the total energy into a part which is the gas, and a part which is the black hole: $E=E_{g}+E_{b h}$. As a crude approximation, we will assume the entropy of the gas is the same that it would be in the absence of the black hole. This may be justified since we are considering small black holes. The total entropy is then

$$
S \sim\left(E_{g} R\right)^{9 / 10}+E_{b h}\left(E_{b h} \ell_{p}^{8}\right)^{1 / 7}
$$

Using $\ell_{p}^{8} \sim R^{8} / N^{2}$, the second term becomes $\left(E_{b h}^{8} R^{8} / N^{2}\right)^{1 / 7}$. Extreming $S$ keeping the total energy fixed yields

$$
E_{g}^{7} E_{b h}^{10} \sim \frac{N^{20}}{R^{17}}
$$

Since the left hand side has a maximum when $E_{g} \sim E_{b h}$, we clearly need $E R>N^{20 / 17}$ in order to have a stable equilibrium. (One can easily check that this is equivalent to our earlier condition $r_{+} / R>N^{-2 / 17}$.) When this condition is satisfied, there are two extrema of the entropy, a local maximum when $E_{b h}>E_{g}$ and a local minimum when $E_{b h}<E_{g}$. When $E R \gg N^{20 / 17}$, the ratio $E_{g} / E_{b h}$ is very small in the maximum entropy configuration. So the net result is that if you fix the total energy, most black holes will 
evaporate slightly and quickly come into equilibrium with their Hawking radiation 1 . This is very similar to earlier studies of a black hole in a box.

A simple check on our result is the following. In order for the black hole to be in stable equilibrium with the radiation, it has to be large enough that it does not evaporate before the radiation has a chance to see the background curvature. In other words, the lifetime of the black hole must be larger than $R$. It is easy to check that in the stable regime, all black holes satisfy this condition. Ignoring the background curvature, the lifetime of a small ten dimensional black hole can be computed from

$$
\frac{d E}{d t} \sim T^{10} r_{+}^{8} \sim \frac{1}{r_{+}^{2}}
$$

Since $E \sim r_{+}^{7} / \ell_{p}^{8}$, the lifetime is $t_{0} \sim r_{+}^{9} / \ell_{p}^{8} \sim N^{2} r_{+}^{9} / R^{8}$. This will be of order $R$ when $r_{+} / R \sim N^{-2 / 9}$. Since $N^{2 / 9} \gg N^{2 / 17}$ for large $N$, this is much smaller than our lower bound for a black hole to be in stable equilibrium. In the context of string theory, another thing we should check is whether the size of the black hole remains bigger than the string scale $\ell_{s}$. Since the smallest stable black hole has $r_{+} / R \sim N^{-2 / 17}$, and $R^{4} \sim g N \ell_{s}^{4}$, we see that $r_{+}$will be larger than $\ell_{s}$ provided $g N>N^{8 / 17}$.

A similar calculation in eleven dimensions shows that black holes have more entropy than a gas of radiation provided $r_{+} / R>\left(\ell_{p} / R\right)^{9 / 19}$ where $\ell_{p}$ is now the eleven dimensional Planck scale.

A second possible misconception concerns the relation between AdS radius and scale size in the CFT. In Poincare coordinates, the AdS metric is simply

$$
d s^{2}=\frac{r^{2}}{R^{2}}\left(-d t^{2}+d x^{i} d x_{i}\right)+\frac{R^{2}}{r^{2}} d r^{2}
$$

Since this metric is invariant under $r \rightarrow \lambda r,\left(t, x^{i}\right) \rightarrow \lambda^{-1}\left(t, x^{i}\right)$, it is often assumed that radial position in AdS is reflected in the scale size of the corresponding excitation in the field theory. This has been checked and is certainly true in many cases, usually involving static configurations. A particularly simple example of this seemed to be a null particle moving radially in AdS. It produces a gravitational shock wave which is reflected in the field theory by a $\left\langle T_{\mu \nu}>\right.$ which is concentrated on the null cone 18. So the expanding excitation in the CFT is correlated with decreasing radial position. However, we should ask what happens if the particle changes its orbit inside AdS. The answer turns out to be

1 This is a more refined version of the discussion in [17. 
that $\left\langle T_{\mu \nu}>\right.$ continues to grow at the speed of light even if the particle stops at some radius $r$ !

This is essentially a consequence of causality: $\left\langle T_{\mu \nu}\right\rangle$ is determined by the asymptotic form of the spacetime metric, and this metric is causally related to the null particle. Letting $\left.z=R^{2} / r, 2.7\right)$ becomes

$$
d s^{2}=\frac{R^{2}}{z^{2}}\left(-d t^{2}+d x_{i} d x^{i}+d z^{2}\right)
$$

A particle initially falling in from $t=x_{i}=z=0$ can only influence fields inside its future light cone $t^{2} \geq x_{i} x^{i}+z^{2}$. A point on the boundary $z=0, t=t^{0}, x_{i}=x_{i}^{0}$ can only be affected by events inside its past light cone $\left(t^{0}-t\right)^{2} \geq\left(x_{i}-x_{i}^{0}\right)^{2}+z^{2}$. Looking at the intersection of these two sets, its clear that the maximum $z$ value for the particle that can affect the asymptotic field at $t^{0}, x_{i}^{0}$ is

$$
z_{\max }=\frac{1}{2}\left[\left(t^{0}\right)^{2}-\left(x_{i}^{0}\right)^{2}\right]^{1 / 2}
$$

which occurs at $t=t^{0} / 2$ and $x_{i}=x_{i}^{0} / 2$. Therefore, as $\left(x_{i}^{0}\right)^{2} \rightarrow\left(t^{0}\right)^{2}$, i.e., one approaches the light cone on the boundary, $z_{\max } \rightarrow 0$. This means that even if the particle, or better yet, a rocket ship, stops at a constant value of $z$ inside AdS, the field will continue to grow along the light cone on the boundary. Of course changing the trajectory inside will produce additional gravitational waves which will result in a change in the expectation value of the stress tensor inside the light cone. But the main lesson is that, in dynamical processes, the size of the disturbance on the boundary is NOT always a measure of the radial position of the particle in the interior. (For further examples of this phenomenon, see [19].)

\section{Quasinormal modes}

A spherical black hole in $A d S_{d}$ is described by

$$
d s^{2}=-f(r) d t^{2}+f(r)^{-1} d r^{2}+r^{2} d \Omega_{d-2}^{2}
$$

where

$$
f(r) \equiv 1+\frac{r^{2}}{R^{2}}-\left(\frac{r_{0}}{r}\right)^{d-3}
$$

The black hole horizon $r=r_{+}$is at the largest zero of $f$. A particle falling into this black hole will produce gravitational waves. A rough estimate for when this radiation reaches 
infinity is just the time it takes for the particle to fall from infinity to the vicinity of the black hole. For a large black hole $r_{+} \gg R$, this is of order $1 / T$ where $T \sim r_{+} / R^{2}$ is the black hole temperature. At late times, this radiation is independent of the details of what fell in. It is described by characteristic oscillations of the black hole geometry known as quasinormal modes [20]. These oscillations are damped and the corresponding quasinormal frequencies are complex. The mode with the smallest imaginary part dominates at late time and gives the timescale for generic perturbations to decay. My student V. Hubeny and I have recently computed these quasinormal frequencies. (For a more complete discussion, see [21].)

The damping time of these oscillations have important implications for the dual CFT. Suppose we start with a large static black hole with temperature $T$. This is described in the field theory by the thermal state 2 with temperature $T$. Perturbing the black hole, corresponds to perturbing this thermal state, and the timescale for the decay of the perturbation is the timescale for the return to thermal equilibrium. This dynamical timescale is extremely difficult to compute directly, but can be done relatively easily using the AdS/CFT correspondence. For simplicity, we considered perturbations described by a real scalar field like the dilaton.

Since a black hole in AdS has two dimensionful parameters $R$ and $r_{+}$, it is not obvious how the quasinormal frequencies $\omega$ will scale as we change the size of the black hole. But for large black holes $r_{+} \gg R$, it turns out that there is an extra symmetry which ensures that $\omega$ will be proportional to the black hole temperature.

Let us decompose the quasinormal frequencies into real and imaginary parts as $\omega=$ $\omega_{R}-i \omega_{I}$. (The sign is chosen so that exponentially decaying modes correspond to $\omega_{I}>0$.) The linear dependence with temperature is clearly shown in fig. 1 and fig. 2 , where $\omega_{I}$ and $\omega_{R}$ respectively are plotted as a function of the temperature for the four, five, and seven dimensional cases. We have set the AdS radius equal to one, so all quantities are measured in units of the AdS radius. The dots, representing the lowest quasinormal mode for each black hole, lie on straight lines through the origin. In fig. 1, the top line corresponds to the $d=4$ case, the middle line is the $d=5$ case, and the bottom line is the $d=7$ case. Explicitly, the lines are given by

$$
\omega_{I}=11.16 T \quad \text { for } d=4
$$

2 For a black hole formed from collapse of a pure state, the CFT state will still be pure, but resemble the thermal state for macroscopic observations. 


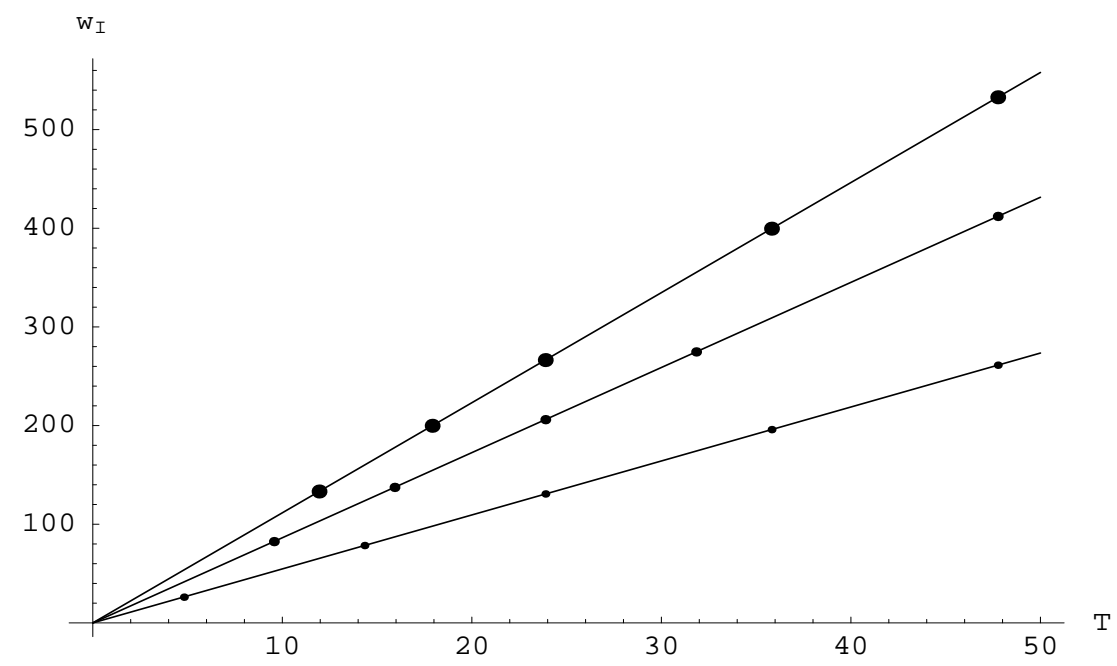

Fig. 1: For large black holes, $\omega_{I}$ is proportional to the temperature. The top line is $d=4$, the middle line is $d=5$ and the bottom line is $d=7$.

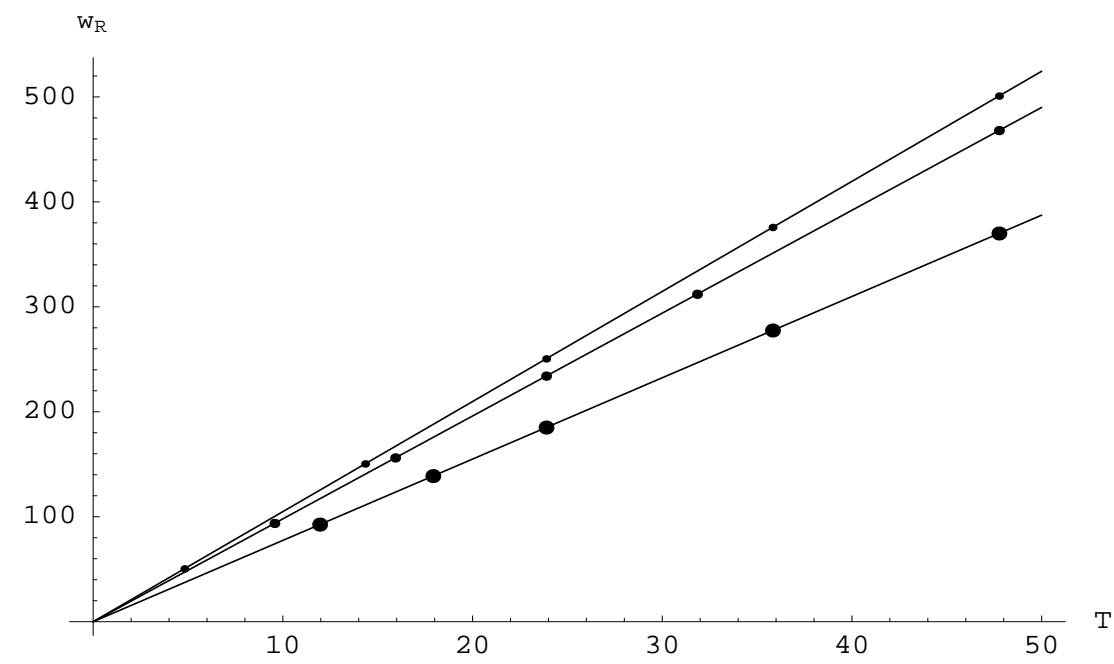

Fig. 2: For large black holes, $\omega_{R}$ is also proportional to the temperature. The top line is now $d=7$, the middle line is $d=5$ and the bottom line is $d=4$.

$$
\begin{array}{ll}
\omega_{I}=8.63 T & \text { for } d=5 \\
\omega_{I}=5.47 T & \text { for } d=7
\end{array}
$$

For smaller black holes, the quasinormal frequencies do not scale with the temperature. This is clearly shown in fig. 3 which plots $\omega_{I}$ as a function of $r_{+}$for $d=4$ black holes with $r_{+} \sim 1$. To a remarkable accuracy, the points continue to lie along a straight line $\omega_{I}=2.66 r_{+}$. The dashed curve represents the continuation of the curve $\omega_{I}=11.16 T$ shown in Fig. 1 to smaller values of $r_{+}$. (For large $r_{+}$these two curves are identical.) It is 


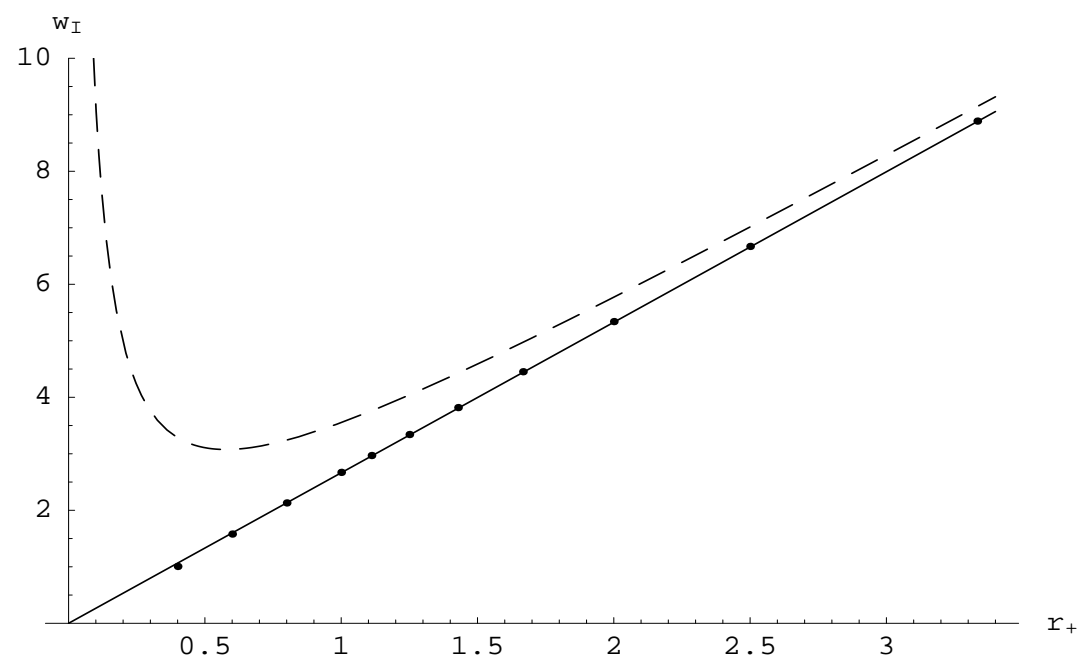

Fig. 3: $\omega_{I}$ for smaller black holes in four dimensions. The solid line is $\omega_{I}=2.66 r_{+}$, and the dashed line is $\omega_{I}=11.16 \mathrm{~T}$.

not yet clear what the significance of this linear relation is for the dual CFT. As we have seen, these black holes are stable if one fixes the total energy, and thus correspond to a class of stable states in the field theory. This linear relation is describing the timescale for the decay of perturbations of these states.

The fact that the quasinormal frequencies do not follow the temperature is very different from small black holes in asymptotically flat spacetimes. In that case, there is only one scale $r_{+}$in the problem and the frequencies must go like $T \sim 1 / r_{+}$. It is different in AdS simply because the boundary conditions at infinity have been changed. It should not be surprising that even for small black holes, the late time behavior of fields is different in AdS than in an asymptotically flat spacetime.

There is a striking similarity between the slope of the line in Fig. 3 and a number that has been computed in a completely different problem. If you study the gravitational collapse of spherically symmetric scalar fields (in four dimensional asymptotically flat spacetimes), one finds that weak waves scatter and go off to infinity while strong waves collapse to form black holes. On the boundary between these two possibilities, there is initial data which collapses to form a 'zero mass black hole', which is really a naked singularity 222. All such initial data approach the same solution, called the critical solution, near the singularity. This critical solution is known to have one unstable modes which grows like $e^{2.67 t}$ [23]. This number is very similar to the slope 2.66 that we found above. Despite the fact that both numbers characterize exponential behavior of spherically symmetric scalar fields in four dimensions, further investigation has failed to find any confirming indications 


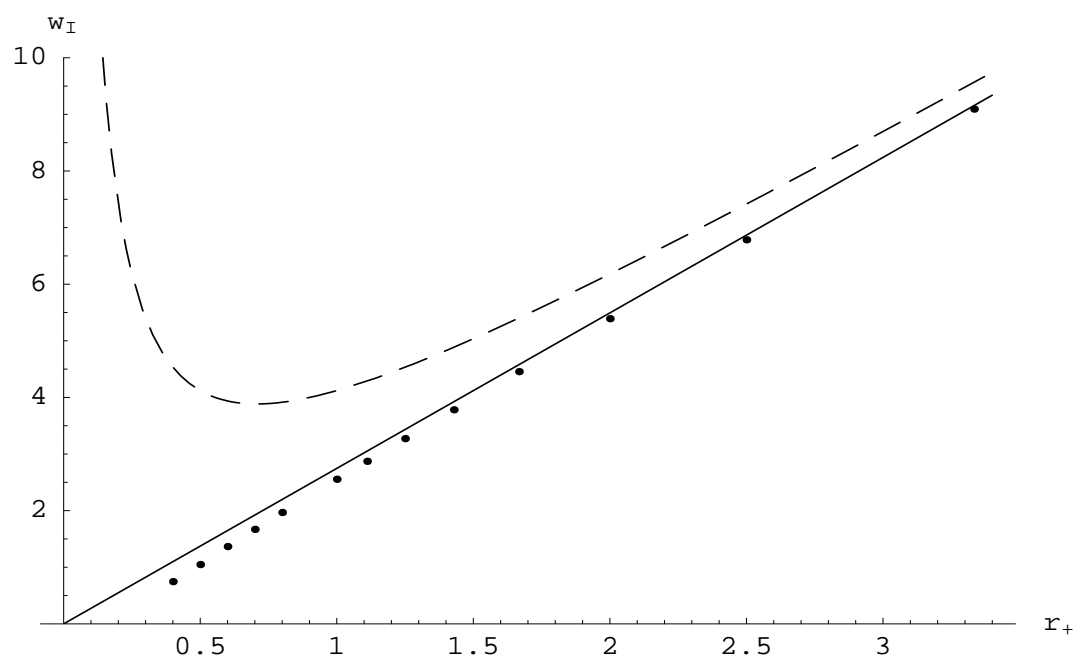

Fig. 4: $\omega_{I}$ for smaller black holes in five dimensions. The solid line is $\omega_{I}=2.75 r_{+}$, and the dashed line is $\omega_{I}=8.63 \mathrm{~T}$.

of a connection between black holes in AdS and black hole critical phenomena. It appears at the moment to be just a numerical coincidence.

One reason for this is that the linear relation does not extend to very small black holes. In fact, since the quasinormal frequencies can be computed to an accuracy much better than the size of the dots in Fig. 3, one can check that the points actually lie slightly off the line. This is shown more clearly in the five dimensional results in Fig. 4. Once again, the dashed curve is the continuation of the curve $\omega_{I}=8.63 \mathrm{~T}$ shown in Fig. 1, and the solid curve is the line $\omega_{I}=2.75 r_{+}$that it approaches asymptotically.

\section{Conclusion}

If I was granted three wishes in the subject of black holes in string theory, they would be:

a) Explain the 3/4 factor relating the weak and strong coupling calculations of the entropy of the near extremal three-brane.

b) Find an exact calculation of the entropy of a Schwarzschild black hole.

c) Understand how (whether?) the usual information loss arguments break down in the evaporation of a small black hole.

We have already discussed (a). The current status of (b) is that there are general arguments which relate uncharged black holes to excited string states, and show that the entropy should be proportional to the horizon area [24,25]. But they are not yet able 
to compute the numerical factor. Finally, we mentioned that in terms of the AdS/CFT correspondence, the evaporation of a small black hole in AdS should be a unitary process in the CFT. But we do not yet understand how the usual semiclassical arguments for information loss break down. This might point toward a possible limitation of the AdS/CFT correspondence, but is more likely just a result of our current lack of understanding of how the CFT describes the spacetime inside the horizon.

\section{Acknowledgements}

I would like to thank the organizers of the Strings '99 conference for a very stimulating meeting. This work was supported in part by NSF grant PHY95-07065. 


\section{References}

[1] G. Horowitz and A. Strominger, Nucl. Phys. B360 (1991) 197.

[2] For a review of some of these solutions, see M. Duff, R. Khuri and J. Lu, Phys. Rep. 259 (1995) 213, hep-th/9412184.

[3] J. Polchinski, Phys. Rev. Lett. 75 (1995) 4724, hep-th/9510017; "TASI Lectures on D-Branes", hep-th/9611050.

[4] S. Gubser, I. Klebanov, and A. Peet, Phys. Rev. D54 (1996) 3915, hep-th/9602135; A. Strominger, unpublished.

[5] See, e.g., R. Dijkgraaf, talk at Strings '99.

[6] A. Strominger and C. Vafa, Phys. Lett. B379 (1996) 99, hep-th/9601029.

[7] C. Callan and J. Maldacena, Nucl. Phys. B472 (1996) 591, hep-th/9602043.

[8] G. Horowitz and A. Strominger, Phys. Rev. Lett. 77 (1996) 2368, hep-th/9602051.

[9] S. Das and S. Mathur, Nucl. Phys. B478 (1996) 561, hep-th/9606185.

[10] J. Maldacena and A. Strominger, Phys. Rev. D55 (1997) 861, hep-th/9609026.

[11] J. Maldacena, Adv. Theor. Math. Phys. 2 (1998) 231, hep-th/9711200.

[12] For a comprehensive review, see O. Aharony, S.S. Gubser, J. Maldacena, H. Ooguri, and Y. Oz, "Large N Field Theories, String Theory and Gravity", hep-th/9905111.

[13] M. Banados, C. Teitelboim, and J. Zanelli, Phys. Rev. Lett. 69 (1992) 1849.

[14] S. Gubser, I. Klebanov, and A. Tseytlin, Nucl. Phys. B534 (1998) 202, hep-th/9805156.

[15] G. Cardoso, B. de Wit, and T. Mohaupt, "Deviations from the Area Law for Supersymmetric Black Holes", hep-th/9904005.

[16] R. Gregory and R. Laflamme, Phys. Rev. Lett, 70 (1993) 2837, hep-th/9301052.

[17] T. Banks, M. Douglas, G. Horowitz, and E. Martinec, "AdS Dynamics from Conformal Field Theory", hep-th/9808016.

[18] G. Horowitz and N. Itzhaki, JHEP 9902 (1999) 010, hep-th/9901012.

[19] V. Balasubramanian and S. Ross, "Holographic Particle Detection", hep-th/9906226.

[20] For a recent review, see K. Kokkotas and B. Schmidt, "Quasi-normal modes of stars and black holes", gr-qc/9909058.

[21] G. Horowitz and V. Hubeny, "Quasinormal Modes of AdS Black Holes and the Approach to Thermal Equilibrium", hep-th/9909056.

[22] M. Choptuik, Phys. Rev. Lett. 70 (1993) 9.

[23] For a review, see C. Gundlach, Adv. Theor. Math. Phys. 2 (1998) 1, gr-qc/9712084.

[24] L. Susskind, "Some Speculations about Black Hole Entropy in String Theory", hep-th/9309145; G. Horowitz and J. Polchinski, Phys. Rev. D55 (1997) 6189, hepth/9612146.

[25] G. Horowitz and J. Polchinski, Phys. Rev. D57 (1998) 2557, hep-th/9707170; T. Damour and G. Veneziano, "Self-gravitating fundamental strings and black-holes", hep-th/9907030. 